\title{
The Amount of Residual Incretin Regulates the Pancreatic $\beta$-cell Function and Glucose Homeostasis
}

\author{
Tatsuya Kondo ${ }^{1}$, Sayaka Kitano ${ }^{1}$, Nobukazu Miyakawa ${ }^{2}$, Takuro Watanabe ${ }^{2}$, Rieko Goto $^{2}$, \\ Miki Sato ${ }^{2}$, Satoko Hanatani ${ }^{2}$, Masaji Sakaguchi ${ }^{2}$, Motoyuki Igata ${ }^{1}$, Junji Kawashima ${ }^{2}$, \\ Hiroyuki Motoshima ${ }^{2}$, Takeshi Matsumura ${ }^{2}$ and Eiichi Araki ${ }^{2}$
}

\begin{abstract}
:
The gastrointestinal tract is considered an important endocrine organ for controlling glucose homeostasis via the production of incretins. A 21-year-old man emergently underwent total colectomy due to severe ulcerative colitis, and overt diabetes became evident. Weekly administration of a glucagon-like peptide (GLP)-1 receptor agonist (RA) dramatically improved his glucose control. Levels of GLP-1 or gastric inhibitory polypeptide (GIP) were low at the baseline in the duodenum and serum of the patient. After 11 months of GLP-1RA treatment, his HbA1c worsened again, and intensive insulin therapy was necessary to control his glucose levels. Our report may explain the significance of residual incretin for maintaining the pancreatic $\beta$ cell function.
\end{abstract}

Key words: colectomy, diabetes, glucagon-like peptide (GLP)-1, gastric inhibitory polypeptide (GIP)

(Intern Med 60: 1433-1442, 2021)

(DOI: 10.2169/internalmedicine.6026-20)

\section{Introduction}

The gastrointestinal tract is considered an important entero-endocrine organ for controlling whole-body glucose homeostasis by producing glucagon-like peptide (GLP)-1, gastric inhibitory polypeptide (GIP) and other molecules $(1,2)$. GLP-1 and GIP are major incretin hormones that are released from the gut during meal ingestion and contribute to the incretin effect by potentiating glucosestimulated insulin secretion and maintain the $\beta$-cell mass (1-5). However, the long-term effects of GLP-1 or GIP on pancreatic $\beta$-cells in humans have yet to be fully elucidated.

Ulcerative colitis (UC) is a chronic inflammatory bowel disease characterized by bloody diarrhea and fecal urgency. Since there are no curative treatments, intractable UC can lead to hospitalization and colectomy (6). Patients with total colectomy were previously found to have a hazard ratio of 1.40 for clinically recorded type 2 diabetes (7). However, the exact mechanisms underlying the development of diabetes in patients with colectomy are not fully understood.

We herein report the clinical case of a patient who received total colectomy due to severe UC and developed diabetes with defects in GLP-1 and GIP production. The patient was initially successfully treated with GLP-1 receptor agonist (RA) but subsequently suffered exacerbation of diabetes with a reduction in insulin secretion.

\section{Materials and Methods}

\section{Study participants}

In addition to the present patient who had diabetes and a history of colectomy $(n=1)$, age-matched non-diabetic, noncolectomy control subjects (young men with a similar body mass index) were recruited for duodenal epithelium sampling $(n=3)$, fecal bacterial 16S rRNA sequencing $(n=3)$ and the measurements of GIP/GLP-1 in serum $(n=5)$.

Written informed consent was obtained from each subject. This study was approved by the institutional review board at Kumamoto University.

${ }^{1}$ Department of Diabetes, Metabolism and Endocrinology, Kumamoto University Hospital, Japan and ${ }^{2}$ Department of Metabolic Medicine, Faculty of Life Sciences, Kumamoto University, Japan 


\section{The GLP-1 and GIP expression in the duodenum}

Duodenal epithelium samples in the middle part of the descending duodenum from the patient and age-matched non-diabetic, non-colectomy control subjects $(n=3)$ were isolated during an upper-gastrointestinal endoscopic examination. Samples were fixed with $10 \%$ natural buffered formalin and then replaced with $30 \%$ sucrose for overnight and embedded with optical cutting temperature (OCT) compound (Sakura Finetek Japan, Tokyo, Japan). Frozen thin-sliced duodenal samples $(10-\mu \mathrm{m}$ thickness) from the patient and control subjects were prepared. Incubation with primary antibodies (anti GLP-1 antibody: ab26278, anti GIP antibody: ab22624; abcam, Cambridge, UK) at 1:100 dilution was performed overnight at $4{ }^{\circ} \mathrm{C}$. After washing, the sections were incubated with secondary antibody conjugated with Alexa Fluor 488 or 555 (Molecular Probes, Eugene, USA) for 1 hour at room temperature. Nuclei were stained with DAPI (1:200 dilution) at room temperature for 5 minutes. After rinsing, the sections were mounted with FluorMounting medium (Diagnostic BioSystems, Pleasanton, USA) and examined with a fluorescence microscope (BZ-9000; Keyence, Osaka, Japan).

\section{The measurement of active GLP-1 and GIP}

Fasted blood samples were isolated using a BD P800 Blood Collection System (BD, Franklin Lakes, USA). Active GLP-1, GIP and glucagon were measured using corresponding enzyme-linked immunosorbent assay (ELISA) kits [Immuno-Biological Laboratories, Tokyo, Japan; GLP-1: human active GLP-1 (\#27784); GIP: human active GIP (\#27201); FUJIFILM Wako Pure Chemical (Osaka, Japan)].

\section{Characterization of gut microbiota}

Fresh fecal samples were collected after defecation and kept under anaerobic conditions. The DNA of isolated bacteria was extracted using a NucleoSpin ${ }^{\circledR}$ Microbial DNA kit (Macherey-Nagel, Düren, Germany), according to the manufacturer's protocol. The extracted DNA was further purified using an AMPureXP (Bechman Coulter, Pasadena, USA). The 16S rRNA gene was amplified from the DNA extracts using a Bacterial 16S rDNA PCR Kit (Takara Bio, Kusatsu, Japan), and the polymerase chain reaction (PCR) products were purified with AMPureXP. The quality of the sequence library was measured using an Agilent 2200 TapeStation. Clustering and phylogenic classification was performed using QIIME (http://qiime.org/) and an Ribosomal Database Project (RDP) classifier (http://rdp.cme.msu.edu/index.jsp), respectively.

\section{Case Report}

A 21-year-old Japanese man started complaining of severe abdominal pain in April 20XX. He underwent total colonoscopy and was diagnosed with UC. His symptoms were severely progressive, so treatment was started with $60 \mathrm{mg}$ prednisolone, $10 \mathrm{mg}$ tacrolimus and 4,000 mg mesalazine. Soon after starting these medications, hyperglycemia of around $300-400 \mathrm{mg} / \mathrm{dL}$ with $\mathrm{HbA} 1 \mathrm{c} 5.2 \%$ and glycated al- bumin (GA) $16.8 \%$ became apparent, indicating that he had not previously had diabetes. In May 20XX, emergency subtotal colectomy (from ileo-cecum to sigmoid) was performed due to the acute development of paralytic ileus. After this intervention, his symptoms were resolved, and the medications were appropriately tapered, but his hyperglycemia persisted. Intensive insulin therapy [lispro $(6,6,6)$ with glargine biosimilar $(0,0,0,8)]$ was therefore implemented, as shown in Fig. 1. At that time, his body mass index was $18.6 \mathrm{~kg} / \mathrm{m}^{2}$.

Throughout the entire clinical course, his body weight and lifestyle habits were not altered. Anti-glutamic acid decarboxylase (GAD) antibody was $<5.0 \mathrm{U} / \mathrm{mL}$, which was confirmed twice and both less than lower limit. Other autoantibodies, such as anti-IA-2 $(<0.6 \mathrm{U} / \mathrm{mL}$; cut-off value 0.6$)$ or anti-ZnT8 $(<10.0 \mathrm{U} / \mathrm{mL}$; cut-off value 15.0$)$, were negative as well. Urinary C-peptide secretion was $52.3 \mu \mathrm{g} /$ day. The C-peptide index (CPI) was 0.7 , and the $\Delta \mathrm{C}$-peptide on a glucagon loading test was $0.4 \mathrm{ng} / \mathrm{mL}$ (Table 1). He possessed HLA-DRB1 1503, suggesting that he might be resistant to type 1 diabetes.

In late June, rectumectomy was performed, and total colectomy was completed, so his UC was finally resolved. After the discontinuation of prednisolone and other agents, insulin therapy was switched to treatment with the dipeptidyl peptidase (DPP)-4 inhibitor vildagliptin, but his HbA1c increased from $6.4 \%$ to $9.5 \%$. Additional treatment of 1,000 $\mathrm{mg}$ metformin and $1 \mathrm{mg}$ glimepiride showed a minor effect on controlling his glucose levels (HbAlc: from $9.5 \%$ to 8.2\%. Fig. 1).

In consideration of the possibility of defective GLP-1 secretion due to total colectomy, weekly GLP-1 RA with 0.75 $\mathrm{mg}$ of dulaglutide was initiated along with the suspension of vildagliptin and glimepiride. After 8 weeks of dulaglutide treatment, his HbA1c dramatically decreased from $8.2 \%$ to $5.9 \%$. After 20 weeks of dulaglutide treatment, HbA1c further decreased to $5.4 \%$ with no adverse events.

Although GLP-1RA dramatically improved his glucose control, this beneficial effect was not sustained. After 11 months of GLP-1RA treatment, his HbA1c worsened again and increased to $11.3 \%$. Intensive insulin therapy [lispro $(12,10,10)$ with glargine biosimilar $(0,0,0,12)]$ was again introduced with GLP-1RA and successfully controlled his $\mathrm{HbA} 1 \mathrm{c}$ at $5.3 \%$. At this point, his urinary C-peptide secretion was $26.8 \mu \mathrm{g} /$ day, his CPI was 0.36 , and his $\Delta \mathrm{C}$-peptide on glucagon loading test was $0.1 \mathrm{ng} / \mathrm{mL}$ (Table 1), indicating that his insulin secretory capacity had decreased to < $50 \%$ since his diabetes had been confirmed. The entire clinical course is shown in Fig. 1.

\section{The analysis of GLP-1 and GIP in the duodenum}

The first dramatic improvement in glucose homeostasis by GLP-1 RA therapy prompted us to investigate the duodenal GLP-1 and GIP expression, serum active GLP-1 and GIP and compositions of gut microbiota. Duodenal samples were isolated from the patient and age-matched non-diabetic, non- 


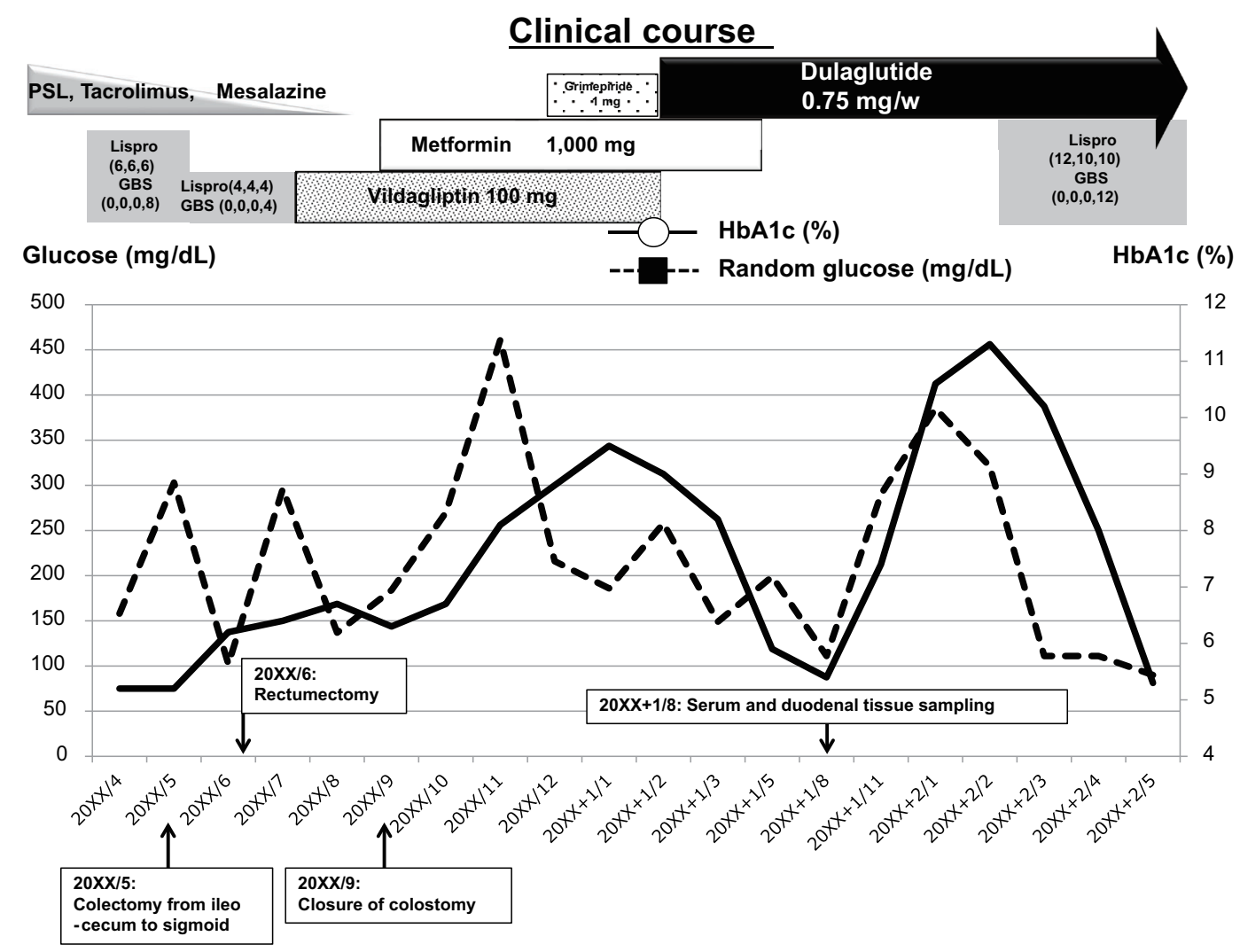

Figure 1. The clinical course of the patient. Medications for the treatment of UC and diabetes, HbA1c (open circle) and fasted glucose (closed square) were indicated. PSL: prednisolone, GBS: glargine biosimilar

\section{patient}
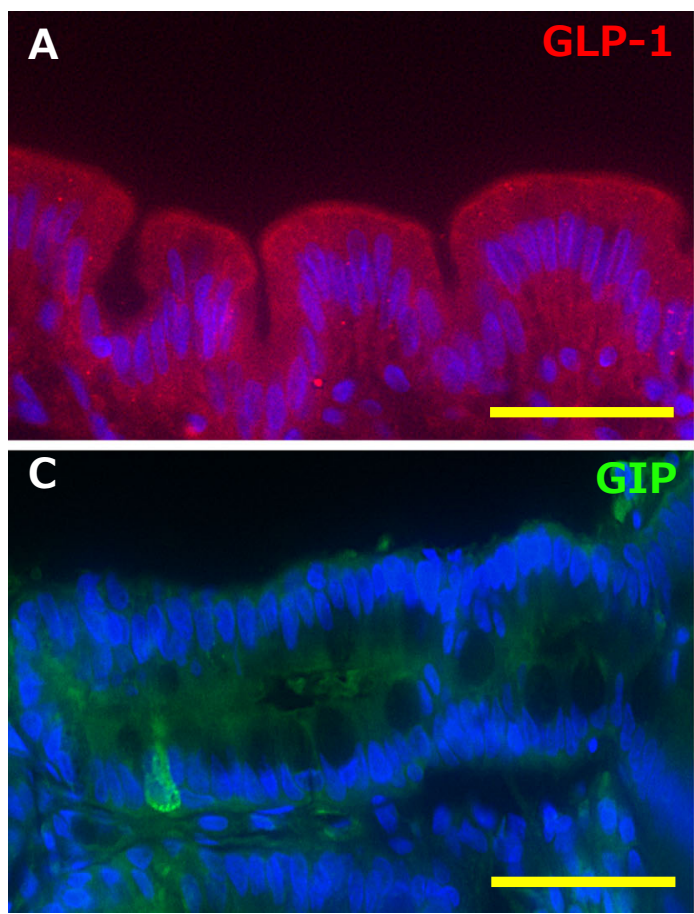

Figure 2. The duodenal expression of GLP-1 and GIP. Frozen sections of duodenal samples from the patient (A, C) and age-matched non-diabetic control subjects $(B, D)$ were fluorescent-immunohistochemically stained for GLP-1 (A, B) or GIP (C, D) with DAPI. The yellow horizontal bar indicates $50 \mu \mathrm{m}$ in length.
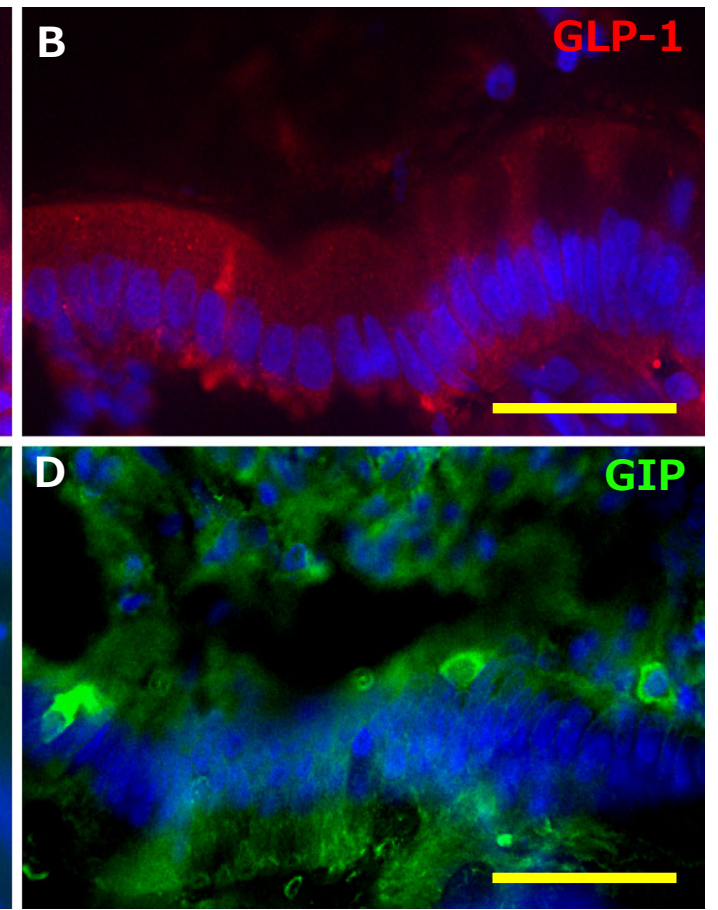

\section{control}


A (cells $\left./ \mathrm{mm}^{2}\right) \quad$ GLP-1 positive cells

15
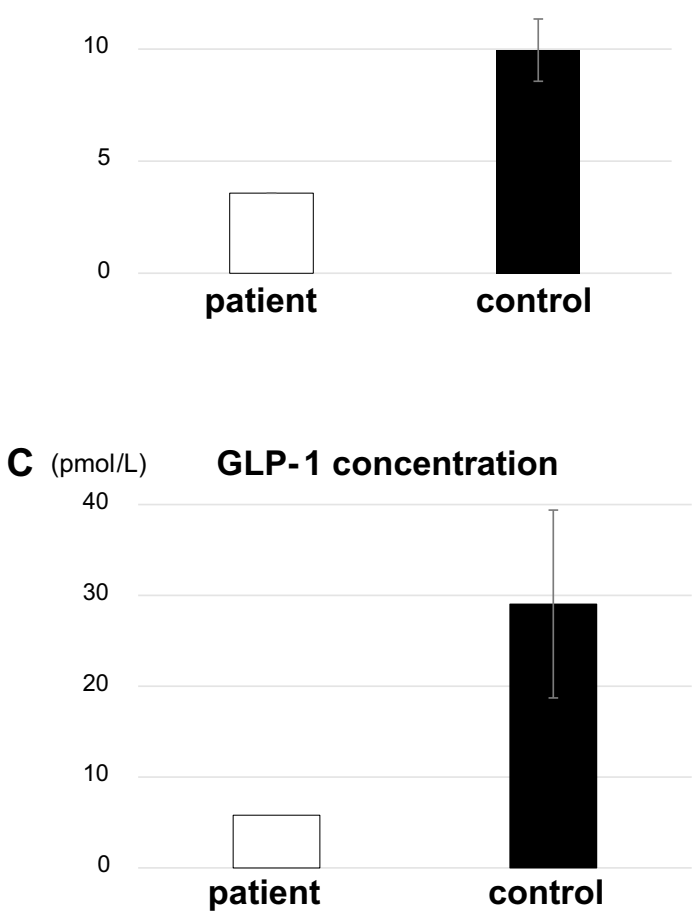

B (cells $\left./ \mathrm{mm}^{2}\right)$ GIP positive cells

30

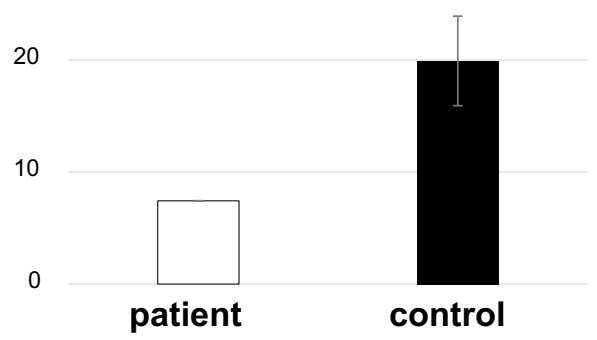

D (pmol/L) GIP concentration

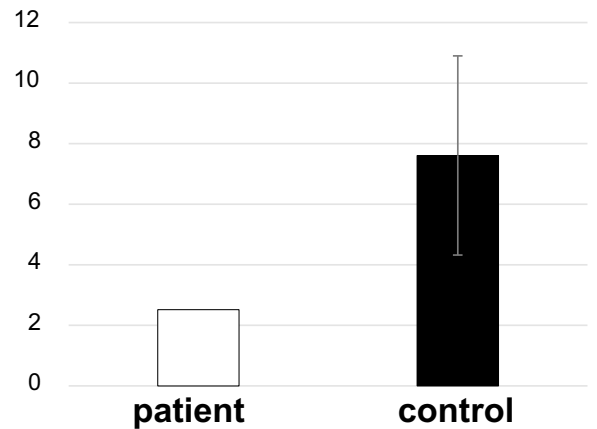

Figure 3. The density (cells/mm²) of GLP-1- or GIP-positive cells (A, B). The numbers of GLP-1- or GIP-positive cells (A, B) were quantified using immunohistochemically stained samples. At least 10 different visual fields were independently quantified. Fasted active GLP-1 or GIP concentration (C, $D)$. Fasted blood samples were isolated from the patient and age-matched non-diabetic control subjects using the BD P800 Blood Collection System. Active GLP-1 or GIP (C, D) was measured using corresponding ELISA kits.

colectomy control subjects $(n=3)$.

The immunohistochemical GLP-1 expression (shown in red; Fig. 2A, B) indicated that GLP-1-positive cells were sparse in the patient but partially positive in the control subjects' duodenum. GIP immunoreactivity (green) in the patient was positive in small populations but more so in the controls (Fig. 2C, D). The quantitative analysis of the density of GLP-1- or GIP-positive cells showed that such cells were rarer in the patient than in the controls (Fig. 3A, B). The fasted serum active GLP-1 level was $5.80 \mathrm{pmol} / \mathrm{L}$ in the patient and $29.05 \pm 10.34 \mathrm{pmol} / \mathrm{L} \quad(\mathrm{n}=5)$ in the controls (Fig. 3C), while the GIP level was $2.52 \mathrm{pmol} / \mathrm{L}$ in the patient and 7.61 $\pm 3.29 \mathrm{pmol} / \mathrm{L}$ in the controls (Fig. 3D).

\section{The analysis of the gut microbiota}

Since the colon possesses a large amount and numerous species of gut microbiota, fecal $16 \mathrm{~S}$ rRNA sequencing was performed in this patient as well as non-diabetic noncolectomized subjects $(n=3)$. Gut microbiota dysbiosis is responsible for GLP-1RA resistance, and Lactobacillaceae numbers were reduced, while Porphyromonadaceae, Clostridiaceae, Peptostreptococaceae and Burkholdericeae were increased in diabetic mice (8). Our results indicated that the numbers of Lactobacillales were comparable among the subjects (Table 4; $0.7 \%$ in the patient and $0.2 \%, 8.2 \%$ and $0.2 \%$ in controls). The proportion of Porphyromonadaceae was $0.0 \%$ in the patient and $1.3 \%, 7.8 \%$ and $3.3 \%$ in the controls (Table 5). The proportion of Clostridiaceae was $16.6 \%$ in the patient and $0.9 \%, 1.4 \%$ and $0.1 \%$ in the controls (Table 5). The proportion of Streptococaceae (family) was $0.5 \%$ in the patient and $0.2 \%, 8.1 \%$ and $0.2 \%$ in the controls (Table 5). The proportion of Burkholderiales (order) was $0.0 \%$ in the patient and $4.2 \%, 0.0 \%$ and $1.5 \%$ in the controls (Table 4).

Increased numbers of Firmicutes and decreased numbers of Bacteroides and Bifidobacterium are associated with obesity and insulin resistance (9). Among the present study, the proportion of Firmicutes (phylum) was $40.8 \%$ in the patient and $44.3 \%, 66.9 \%$ and $69.8 \%$ in the controls (Table 2). The proportion of Bacteroidetes (phylum) was $31.7 \%$ in the patient and $41.6 \%, 31.4 \%$ and $20.9 \%$ in the controls (Table 2). The proportion of Bifidobacterium (genus) was $25.8 \%$ in the patient and $2.0 \%, 0.1 \%$ and $3.7 \%$ in the controls (Table 6).

\section{Discussion}

An increased risk of clinically recorded type 2 diabetes had been reported among patients who underwent total 
Table 1. The Changes in Fasted Biochemical Parameters during the Clinical Course of the Patient.

\begin{tabular}{|c|c|c|c|c|}
\hline & On admission & After colectomy & GLP-1RA failure & Most recent \\
\hline & May 20XX & Jan $20 X X+1$ & Feb $20 X X+1$ & Jul $20 X X+1$ \\
\hline $\mathrm{TP}(\mathrm{g} / \mathrm{dL})$ & 6.4 & 7.8 & 7.4 & 7.5 \\
\hline $\mathrm{Alb}(\mathrm{g} / \mathrm{dL})$ & 2.4 & 4.9 & 4.6 & 4.7 \\
\hline $\mathrm{Na}(\mathrm{mEq} / \mathrm{L})$ & 129 & 138 & 134 & 143 \\
\hline $\mathrm{K}(\mathrm{mEq} / \mathrm{L})$ & 4.9 & 4.2 & 4.8 & 4.3 \\
\hline $\mathrm{Cl}(\mathrm{mEq} / \mathrm{L})$ & 101 & 102 & 98 & 103 \\
\hline $\mathrm{Ca}(\mathrm{mg} / \mathrm{dL})$ & 7.8 & 9.8 & 10.1 & 10.1 \\
\hline IP (mg/dL) & 1.7 & 4.2 & 4.2 & 5.2 \\
\hline $\mathrm{Mg}(\mathrm{mg} / \mathrm{dL})$ & 2.4 & 1.8 & 1.7 & \\
\hline $\mathrm{UA}(\mathrm{mg} / \mathrm{dL})$ & 1.8 & 2.7 & 2.7 & 3.4 \\
\hline BUN (mg/dL) & 10.8 & 14.4 & 18.8 & 18.1 \\
\hline Creatinine (mg/dL) & 0.77 & 0.79 & 0.75 & 0.73 \\
\hline $\mathrm{eGFR}\left(\mathrm{mL} / \mathrm{min} / 1.73 \mathrm{~m}^{2}\right)$ & $>90$ & $>90$ & $>90$ & $>90$ \\
\hline Glucose (mg/dL) & 177 & 186 & 321 & 111 \\
\hline $\mathrm{HbA1c}(\%)$ & 5.2 & 9.5 & 11.3 & 5.3 \\
\hline GA $(\%)$ & 16.8 & 38.6 & 44.3 & 16.7 \\
\hline T-Bil (mg/dL) & 1.1 & 2.7 & 2.2 & 2.2 \\
\hline AST (U/L) & 17 & 26 & 19 & 17 \\
\hline $\operatorname{ALT}(\mathrm{U} / \mathrm{L})$ & 24 & 14 & 21 & 20 \\
\hline $\mathrm{LD}(\mathrm{U} / \mathrm{L})$ & 249 & 273 & 289 & 216 \\
\hline CK (U/L) & 45 & 155 & 92 & 80 \\
\hline $\mathrm{Fe}(\mu \mathrm{g} / \mathrm{dL})$ & 34 & 101 & 84 & 94 \\
\hline UIBC $(\mu \mathrm{g} / \mathrm{dL})$ & 202 & 310 & 234 & 273 \\
\hline $\mathrm{T}-\mathrm{CHO}(\mathrm{mg} / \mathrm{dL})$ & 128 & 124 & 151 & 163 \\
\hline $\mathrm{TG}(\mathrm{mg} / \mathrm{dL})$ & 56 & 76 & 112 & 57 \\
\hline HDL-C (mg/dL) & 63 & 49 & 61 & 72 \\
\hline LDL-C (mg/dL) & 60 & 67 & 77 & 86 \\
\hline T-keton (mmol/L) & & 50.4 & 136.5 & 31.2 \\
\hline $\operatorname{AcAc}(\mathrm{mmol} / \mathrm{L})$ & & 22.7 & 65.1 & 19.6 \\
\hline 3-OHBA (mmol/L) & & 27.7 & 71.4 & 11.6 \\
\hline CRP & 32.18 & 0.07 & 0.33 & 0.11 \\
\hline $\mathrm{GH}(\mathrm{ng} / \mathrm{mL})$ & & 0.4 & 4.55 & \\
\hline IGF-1 (ng/mL) & & 233 & 117 & \\
\hline ACTH (pg/mL) & 2.65 & 31.49 & 40.91 & \\
\hline cortisol ( $\mu \mathrm{g} / \mathrm{dL})$ & 0.7 & 9.5 & 17.4 & \\
\hline C-peptide (ng/mL) & 1.4 & 1.3 & & 0.4 \\
\hline urine-C-peptide (mg/day) & & 52.3 & 26.8 & \\
\hline C-peptide index & & 0.7 & & 0.36 \\
\hline$\Delta \mathrm{C}$-peptide on glucagon load & & 0.4 & & 0.1 \\
\hline $\mathrm{WBC}(/ \mu \mathrm{L})$ & 31,900 & 8,000 & 8,200 & 8,900 \\
\hline Baso (\%) & 0.4 & 0.4 & 0.4 & 0.4 \\
\hline $\operatorname{Eosin}(\%)$ & 0.1 & 0.5 & 1.1 & 0.4 \\
\hline Neut $(\%)$ & 91.8 & 70.5 & 65.6 & 77.5 \\
\hline Lymph (\%) & 4.4 & 23.1 & 29.6 & 17.4 \\
\hline Mono (\%) & 3.3 & 5.5 & 3.3 & 4.3 \\
\hline $\mathrm{RBC}\left(\times 10^{6} / \mu \mathrm{L}\right)$ & 4.22 & 5.81 & 5.65 & 5.28 \\
\hline $\mathrm{Hb}(\mathrm{g} / \mathrm{dL})$ & 12.6 & 15.3 & 16.6 & 15.3 \\
\hline Hct $(\%)$ & 35.2 & 44.8 & 46.2 & 44.6 \\
\hline $\operatorname{PLT}\left(\times 10^{6} / \mu \mathrm{L}\right)$ & 517 & 330 & 336 & 321 \\
\hline
\end{tabular}

TP: thyroid peroxidase, Alb: albumin, BUN: blood urea nitrogen, eGFR: estimated glomerular filtration rate, HbAlc: hemoglobin A1c, GA: glycoalbumin, AST: aspartate transaminase, ALT: alanine aminotransferase, CK: creatine kinase, UIBC: unsaturated iron binding capacity, T-CHO: total cholesterol, HDL-C: high-density lipoprotein cholesterol, LDL: low-density lipoprotein cholesterol, AcAc: acetoacetic acid, 3-OHBA: 3-hydorxybutyric acid, CRP: C-reactive protein, IGF-1: insulin-like growth factor-1, ACTH: adrenocorticotropic hormone, WBC: white blood cell, RBC: red blood cell, Hb: hemoglobin, Hct: hematocrit, Plt: platelet 
Table 2. Taxonomic Summary: Phylum.

\begin{tabular}{lcccc}
\hline \multicolumn{1}{c}{ Taxonomy } & Patient & Control-1 & Control-2 & Control-2 \\
\hline k__Bacteria;p__Actinobacteria & $25.8 \%$ & $6.1 \%$ & $0.4 \%$ & $7.2 \%$ \\
k__Bacteria;p__Bacteroidetes & $31.7 \%$ & $41.6 \%$ & $31.4 \%$ & $20.9 \%$ \\
k__Bacteria;p__Firmicutes & $40.8 \%$ & $44.3 \%$ & $66.9 \%$ & $69.8 \%$ \\
k__Bacteria;p__Fusobacteria & $0.0 \%$ & $3.3 \%$ & $0.4 \%$ & $0.5 \%$ \\
k_Bacteria;p__Proteobacteria & $1.6 \%$ & $4.7 \%$ & $1.0 \%$ & $1.6 \%$ \\
\hline
\end{tabular}

Table 3. Taxonomic Summary: Class.

\begin{tabular}{|c|c|c|c|c|}
\hline Taxonomy & Patient & Control-1 & Control-2 & Control-2 \\
\hline k__Bacteria;p_Actinobacteria;c_Actinobacteria & $25.8 \%$ & $2.0 \%$ & $0.1 \%$ & $3.7 \%$ \\
\hline k__Bacteria;p_Actinobacteria;c_Coriobacteriia & $0.0 \%$ & $4.1 \%$ & $0.3 \%$ & $3.5 \%$ \\
\hline k__Bacteria;p__Bacteroidetes;c__Bacteroidia & $31.7 \%$ & $41.6 \%$ & $31.4 \%$ & $20.9 \%$ \\
\hline k__Bacteria;p_Firmicutes;c__Bacilli & $6.3 \%$ & $0.2 \%$ & $8.2 \%$ & $0.2 \%$ \\
\hline k__Bacteria;p__Firmicutes;c__Clostridia & $26.3 \%$ & $43.1 \%$ & $55.0 \%$ & $61.2 \%$ \\
\hline k__Bacteria;p_Firmicutes;c_Erysipelotrichi & $8.2 \%$ & $1.1 \%$ & $3.7 \%$ & $8.4 \%$ \\
\hline k__Bacteria;p__Fusobacteria;c__Fusobacteriia & $0.0 \%$ & $3.3 \%$ & $0.4 \%$ & $0.5 \%$ \\
\hline k__Bacteria;p_Proteobacteria;c__Betaproteobacteria & $0.0 \%$ & $4.2 \%$ & $0.0 \%$ & $1.5 \%$ \\
\hline k__Bacteria;p__Proteobacteria;c__Deltaproteobacteria & $0.0 \%$ & $0.0 \%$ & $0.0 \%$ & $0.1 \%$ \\
\hline k__Bacteria;p_Proteobacteria;c__Gammaproteobacteria & $1.6 \%$ & $0.6 \%$ & $1.0 \%$ & $0.0 \%$ \\
\hline
\end{tabular}

Table 4. Taxonomic Summary: Order.

\begin{tabular}{|c|c|c|c|c|}
\hline Taxonomy & Patient & Control-1 & Control-2 & Control-2 \\
\hline k__Bacteria;p__Actinobacteria;c_Actinobacteria;o_Actinomycetales & $0.0 \%$ & $0.0 \%$ & $0.0 \%$ & $0.0 \%$ \\
\hline k__Bacteria;p__Actinobacteria;c__Actinobacteria;o__Bifidobacteriales & $25.8 \%$ & $2.0 \%$ & $0.1 \%$ & $3.7 \%$ \\
\hline 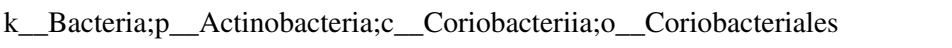 & $0.0 \%$ & $4.1 \%$ & $0.3 \%$ & $3.5 \%$ \\
\hline k__Bacteria;p__Bacteroidetes;c__Bacteroidia;o__Bacteroidales & $31.7 \%$ & $41.6 \%$ & $31.4 \%$ & $20.9 \%$ \\
\hline k__Bacteria;p__Firmicutes;c__Bacilli;o__Bacillales & $5.6 \%$ & $0.0 \%$ & $0.0 \%$ & $0.0 \%$ \\
\hline k__Bacteria;p_Firmicutes;c__Bacilli;o__Lactobacillales & $0.7 \%$ & $0.2 \%$ & $8.2 \%$ & $0.2 \%$ \\
\hline k__Bacteria;p__Firmicutes;c__Clostridia;o_Clostridiales & $26.3 \%$ & $43.1 \%$ & $55.0 \%$ & $61.2 \%$ \\
\hline k__Bacteria;p__Firmicutes;c__Erysipelotrichi;o_Erysipelotrichales & $8.2 \%$ & $1.1 \%$ & $3.7 \%$ & $8.4 \%$ \\
\hline k__Bacteria;p__Fusobacteria;c__Fusobacteriia;o_Fusobacteriales & $0.0 \%$ & $3.3 \%$ & $0.4 \%$ & $0.5 \%$ \\
\hline k__Bacteria;p__Proteobacteria;c__Betaproteobacteria;o__Burkholderiales & $0.0 \%$ & $4.2 \%$ & $0.0 \%$ & $1.5 \%$ \\
\hline k__Bacteria;p__Proteobacteria;c__Deltaproteobacteria;o__Desulfovibrionales & $0.0 \%$ & $0.0 \%$ & $0.0 \%$ & $0.1 \%$ \\
\hline k__Bacteria;p__Proteobacteria;c__Gammaproteobacteria;o__Enterobacteriales & $1.5 \%$ & $0.6 \%$ & $1.0 \%$ & $0.0 \%$ \\
\hline k__Bacteria;p__Proteobacteria;c__Gammaproteobacteria;o__Pasteurellales & $0.1 \%$ & $0.0 \%$ & $0.0 \%$ & $0.0 \%$ \\
\hline
\end{tabular}

colectomy, amounting to a 1.4-fold increased incidence (7).

It is widely accepted that GIP-producing $\mathrm{K}$ cells are densely populated in the small intestine, while GLP-1producing $\mathrm{L}$ cells are largely distributed in the colon in a reciprocally gradational manner $(1,2,10)$. UC patients with colectomy show a slowed release of GLP-1 in response to the intake of glucose $(11,12)$. The insulin and GIP peak levels are higher in UC patients who have undergone colectomy than non-colectomized controls (11). Thus, glucose homeostasis in colectomized patients with a reduced GLP-1 production may be compensated by an enhanced GIP release.

Given the above, we initially speculated that the present post-colectomy patient might still have certain amounts of incretins in the residual small intestine, but DPP-4 inhibition did not contribute markedly to controlling his glucose levels.
Since metformin was suggested to potentially have beneficial effects on the gut microbiota (13), 1,000 mg of metformin was added, but his glucose levels failed to be controlled. A major defect of GLP-1 production was therefore suspected, and weekly GLP-1 RA was initiated, which dramatically improved his glucose levels.

An immuno-histochemical evaluation of the GLP-1- and GIP-producing cells in the residual duodenum revealed that the patient possessed almost no GLP-1-positive cells and had only a few GIP-positive cells compared with control subjects. Consistent with these data, the serum active levels of GLP-1 and GIP in the patient were lower than those in the controls. The defective GLP-1 release in this patient with colectomy may have been caused by the loss of colonic GLP-1-producing entero-endocrine cells. Thus, his incretin production may have been lower at the baseline, with his 
Table 5. Taxonomic Summary: Family.

\begin{tabular}{|c|c|c|c|c|}
\hline Taxonomy & Patient & Control-1 & Control-2 & Control-2 \\
\hline k__Bacteria;p_Actinobacteria;c_Actinobacteria;o__Actinomycetales;f__Micrococcaceae & $0.0 \%$ & $0.0 \%$ & $0.0 \%$ & $0.0 \%$ \\
\hline k__Bacteria;p_Actinobacteria;c_Actinobacteria;o_Bifidobacteriales;f__Bifidobacteriaceae & $25.8 \%$ & $2.0 \%$ & $0.1 \%$ & $3.7 \%$ \\
\hline k__Bacteria;p__Bacteroidetes;c__Bacteroidia;o__Bacteroidales;f__Bacteroidaceae & $31.7 \%$ & $40.3 \%$ & $22.9 \%$ & $17.5 \%$ \\
\hline k__Bacteria;p__Bacteroidetes;c_Bacteroidia;o__Bacteroidales;f__Porphyromonadaceae & $0.0 \%$ & $1.3 \%$ & $7.8 \%$ & $3.3 \%$ \\
\hline k__Bacteria;p__Bacteroidetes;c_Bacteroidia;o__Bacteroidales;f__Rikenellaceae & $0.0 \%$ & $0.0 \%$ & $0.7 \%$ & $0.0 \%$ \\
\hline k__Bacteria;p__Bacteroidetes;c__Bacteroidia;o__Bacteroidales;f__[Barnesiellaceae] & $0.0 \%$ & $0.0 \%$ & $0.0 \%$ & $0.0 \%$ \\
\hline k__Bacteria;p__Bacteroidetes;c__Bacteroidia;o__Bacteroidales;f__[Odoribacteraceae] & $0.0 \%$ & $0.0 \%$ & $0.0 \%$ & $0.1 \%$ \\
\hline k__Bacteria;p__Firmicutes;c__Bacilli;o__Bacillales;f__Bacillaceae & $5.6 \%$ & $0.0 \%$ & $0.0 \%$ & $0.0 \%$ \\
\hline k__Bacteria;p__Firmicutes;c__Bacilli;o__Lactobacillales;f__Carnobacteriaceae & $0.0 \%$ & $0.0 \%$ & $0.0 \%$ & $0.0 \%$ \\
\hline k__Bacteria;p_Firmicutes;c_Bacilli;o__Lactobacillales;f_Enterococcaceae & $0.2 \%$ & $0.0 \%$ & $0.0 \%$ & $0.0 \%$ \\
\hline k__Bacteria;p_Firmicutes;c__Bacilli;o__Lactobacillales;f__Leuconostocaceae & $0.0 \%$ & $0.0 \%$ & $0.0 \%$ & $0.0 \%$ \\
\hline k__Bacteria;p__Firmicutes;c__Bacilli;o__Lactobacillales;f__Streptococcaceae & $0.5 \%$ & $0.2 \%$ & $8.1 \%$ & $0.2 \%$ \\
\hline k__Bacteria;p_Firmicutes;c_Clostridia;o__Clostridiales;f__ & $0.0 \%$ & $0.0 \%$ & $0.0 \%$ & $0.0 \%$ \\
\hline k__Bacteria;p_Firmicutes;c__Clostridia;o_Clostridiales;f__Clostridiaceae & $16.6 \%$ & $0.9 \%$ & $1.4 \%$ & $0.1 \%$ \\
\hline k__Bacteria;p__Firmicutes;c__Clostridia;o__Clostridiales;f__Lachnospiraceae & $0.5 \%$ & $22.8 \%$ & $47.0 \%$ & $43.1 \%$ \\
\hline k__Bacteria;p__Firmicutes;c__Clostridia;o__Clostridiales;f__Ruminococcaceae & $0.0 \%$ & $1.5 \%$ & $3.7 \%$ & $12.7 \%$ \\
\hline k__Bacteria;p__Firmicutes;c_Clostridia;o__Clostridiales;f__Veillonellaceae & $9.1 \%$ & $17.9 \%$ & $2.9 \%$ & $4.3 \%$ \\
\hline k__Bacteria;p_Firmicutes;c__Clostridia;o__Clostridiales;f__[Mogibacteriaceae] & $0.0 \%$ & $0.0 \%$ & $0.0 \%$ & $0.0 \%$ \\
\hline k__Bacteria;p__Firmicutes;c__Erysipelotrichi;o_Erysipelotrichales;f_Erysipelotrichaceae & $8.2 \%$ & $1.1 \%$ & $3.7 \%$ & $8.4 \%$ \\
\hline k__Bacteria;p__Fusobacteria;c__Fusobacteriia;o__Fusobacteriales;f__Fusobacteriaceae & $0.0 \%$ & $3.3 \%$ & $0.4 \%$ & $0.5 \%$ \\
\hline k__Bacteria;p_Proteobacteria;c_Betaproteobacteria;o__Burkholderiales;f__Alcaligenaceae & $0.0 \%$ & $4.2 \%$ & $0.0 \%$ & $1.5 \%$ \\
\hline k__Bacteria;p__Proteobacteria;c__Deltaproteobacteria;o__Desulfovibrionales;f__Desulfovibrionaceae & $0.0 \%$ & $0.0 \%$ & $0.0 \%$ & $0.1 \%$ \\
\hline k__Bacteria;p__Proteobacteria;c__Gammaproteobacteria;o_Enterobacteriales;f__Enterobacteriaceae & $1.5 \%$ & $0.6 \%$ & $1.0 \%$ & $0.0 \%$ \\
\hline k__Bacteria;p__Proteobacteria;c__Gammaproteobacteria;o__Pasteurellales;f__Pasteurellaceae & $0.1 \%$ & $0.0 \%$ & $0.0 \%$ & $0.0 \%$ \\
\hline
\end{tabular}

diabetes suddenly triggered by the elimination of his GLP-1 due to total colectomy.

During the clinical course, his glucose control again worsened under single GLP-1RA therapy. Further intensive insulin therapy was thus necessary to control his hyperglycemia, and his insulin-producing capability was found to have been reduced by almost 50\% compared with that at the development of his diabetes. Since both his GLP-1 and GIP production appeared to be lower than normal by nature, GLP-1 suspension alone may not have been enough to maintain his pancreatic $\beta$-cell mass and/or function. Lower GIP levels may have contributed to a reduction in his $\beta$-cell integrity despite the continuous administration of supraphysiological GLP-1. In this context, sudden GLP-1 loss due to colectomy may have immediately caused diabetes (which was able to be restored by GLP-1RA), but long-term GIP loss might gradually further impair his pancreatic $\beta$-cell function. Indeed, GLP-1 and GIP double-knockout mice exhibited significantly impaired glucose excursion with decreased insulin secretion (4).

At the onset of diabetes in this patient, his CPI was 0.7 and his $\Delta \mathrm{C}$-peptide on the glucagon loading test was $0.4 \mathrm{ng} /$ $\mathrm{mL}$. The efficacy of GLP-1RA monotherapy or sulfonylurea combination depends on the remaining $\beta$-cell function (14). A $\Delta$ C-peptide on the glucagon loading test of $2.34 \mathrm{ng} / \mathrm{mL}$ or CPI of 1.86 have been reported as cut-off values for a longer therapeutic durability of initial GLP-1 RA in Japa- nese populations (15), suggesting that this particular case already possessed a reduced insulin secretory capacity at the initiation of GLP-1RA. The deterioration of the glucose control with GLP-1RA in the short-term in this patient may have been due to his reduced remaining $\beta$-cell function.

Glucose homeostasis in colectomized patients with a reduction in GLP-1 may be compensated for by enhanced GIP release (11). Thus, this compensatory mechanism may not have been sufficient to maintain the $\beta$-cell function in this particular case. Indeed, GIP seems to have been quantitatively the most important incretin, particularly with regard to insulin secretion (5). Why this particular patient possessed fewer L- and K-cells by nature is unclear. There was no family history of diabetes, but possible mechanisms underlying the reduced density of these cells include 1) developmental insufficiency, 2) down-regulation and/or 3) dedifferentiation of enteroendocrine cells. Some transcription factors, such as Rfx6, Arx, Pax4 and Is11, are reportedly important for triggering the differentiation of peptidergic enteroendocrine cells, such as GIP- and GLP-1-secreting cells (16). However, such transcription factors were not evaluated in this patient, so detailed molecular approaches are warranted to clarify the mechanisms underlying the development and/or maintenance of $\mathrm{L}$ - and $\mathrm{K}$-cells.

The bacterial load increases along the length of the colon (17), and colectomies involving the left part of the colon are therefore likely lead to the removal of a larger part of 
Table 6. Taxonomic Summary: Genus.

\begin{tabular}{|c|c|c|c|c|}
\hline Taxonomy & Patient & $\begin{array}{c}\text { Control } \\
-1\end{array}$ & $\begin{array}{c}\text { Control } \\
-2\end{array}$ & $\begin{array}{c}\text { Control } \\
-2\end{array}$ \\
\hline k_Bacteria;p_Actinobacteria;c_Actinobacteria;o_Actinomycetales;f__Micrococcaceae;g_Rothia & $0.0 \%$ & $0.0 \%$ & $0.0 \%$ & $0.0 \%$ \\
\hline k__Bacteria;p_Actinobacteria;c_Actinobacteria;o__Bifidobacteriales;f__Bifidobacteriaceae;g_Bifidobacterium & $25.8 \%$ & $2.0 \%$ & $0.1 \%$ & $3.7 \%$ \\
\hline k_Bacteria;p_Actinobacteria;c__Coriobacteriia;o_CCoriobacteriales;f__Coriobacteriaceae;g_Collinsella & $0.0 \%$ & $3.6 \%$ & $0.0 \%$ & $3.4 \%$ \\
\hline k_Bacteria;p_Actinobacteria;c__Coriobacteriia;o__Coriobacteriales;f_Coriobacteriaceae;g_Eggerthella & $0.0 \%$ & $0.5 \%$ & $0.3 \%$ & $0.0 \%$ \\
\hline k_Bacteria;p_Actinobacteria;c__Coriobacteriia;o__Coriobacteriales;f__Coriobacteriaceae;g_Slackia & $0.0 \%$ & $0.0 \%$ & $0.0 \%$ & $0.1 \%$ \\
\hline k_Bacteria;p__Bacteroidetes;c__Bacteroidia;o__Bacteroidales;f_Porphyromonadaceae;g_Parabacteroides & $0.0 \%$ & $1.3 \%$ & $7.8 \%$ & $3.3 \%$ \\
\hline k_Bacteria;p__Bacteroidetes;c__Bacteroidia;o__Bacteroidales;f_Rikenellaceae;g__ & $0.0 \%$ & $0.0 \%$ & $0.7 \%$ & $0.0 \%$ \\
\hline k__Bacteria;p__Bacteroidetes;c_Bacteroidia;o__Bacteroidales;f__[Barnesiellaceae];g_ & $0.0 \%$ & $0.0 \%$ & $0.0 \%$ & $0.0 \%$ \\
\hline k__Bacteria;p__Bacteroidetes;c__Bacteroidia;o__Bacteroidales;f__[Odoribacteraceae];g_Butyricimonas & $0.0 \%$ & $0.0 \%$ & $0.0 \%$ & $0.1 \%$ \\
\hline k_Bacteria;p__Bacteroidetes;c__Bacteroidia;o__Bacteroidales;f__[Odoribacteraceae];g_Odoribacter & $0.0 \%$ & $0.0 \%$ & $0.0 \%$ & $0.0 \%$ \\
\hline k_Bacteria;p__Firmicutes;c__Bacilli;o__Bacillales;f__Bacillaceae;g_Bacillus & $5.6 \%$ & $0.0 \%$ & $0.0 \%$ & $0.0 \%$ \\
\hline k__Bacteria;p__Firmicutes;c__Bacilli;o__Lactobacillales;f_Enterococcaceae;Other & $0.2 \%$ & $0.0 \%$ & $0.0 \%$ & $0.0 \%$ \\
\hline k_Bacteria;p__Firmicutes;c__Bacilli;o__Lactobacillales;f_LLeuconostocaceae;Other & $0.0 \%$ & $0.0 \%$ & $0.0 \%$ & $0.0 \%$ \\
\hline k_Bacteria;p_Firmicutes;c__Bacilli;o__Lactobacillales;f__Streptococcaceae;g__Lactococcus & $0.0 \%$ & $0.0 \%$ & $0.1 \%$ & $0.0 \%$ \\
\hline k__Bacteria;p_Firmicutes;c__Bacilli;o__Lactobacillales;f_Streptococcaceae;g__Streptococcus & $0.5 \%$ & $0.2 \%$ & $8.1 \%$ & $0.2 \%$ \\
\hline k__Bacteria;p_Firmicutes;c__Clostridia;o__Clostridiales;Other;Other & $0.1 \%$ & $0.0 \%$ & $0.0 \%$ & $0.9 \%$ \\
\hline k__Bacteria;p_Firmicutes;c__Clostridia;o__Clostridiales;f_;g__ & $0.0 \%$ & $0.0 \%$ & $0.0 \%$ & $0.0 \%$ \\
\hline k__Bacteria;p__Firmicutes;c__Clostridia;o__Clostridiales;f_CClostridiaceae;Other & $1.4 \%$ & $0.0 \%$ & $0.0 \%$ & $0.0 \%$ \\
\hline k__Bacteria;p__Firmicutes;c__Clostridia;o__Clostridiales;f_CClostridiaceae;g__Clostridium & $6.1 \%$ & $0.1 \%$ & $0.0 \%$ & $0.1 \%$ \\
\hline k__Bacteria;p__Firmicutes;c__Clostridia;o__Clostridiales;f_Clostridiaceae;g_SMB53 & $9.1 \%$ & $0.7 \%$ & $1.4 \%$ & $0.0 \%$ \\
\hline k__Bacteria;p__Firmicutes;c__Clostridia;o__Clostridiales;f__Lachnospiraceae;Other & $0.0 \%$ & $0.2 \%$ & $1.1 \%$ & $12.3 \%$ \\
\hline k__Bacteria;p_Firmicutes;c__Clostridia;o__Clostridiales;f_LLachnospiraceae;g__ & $0.0 \%$ & $0.2 \%$ & $4.2 \%$ & $7.3 \%$ \\
\hline k__Bacteria;p_Firmicutes;c__Clostridia;o__Clostridiales;f_L_achnospiraceae;g_Anaerostipes & $0.0 \%$ & $0.0 \%$ & $0.0 \%$ & $0.1 \%$ \\
\hline k_Bacteria;p_Firmicutes;c__Clostridia;o__Clostridiales;f_Lachnospiraceae;g_Blautia & $0.0 \%$ & $4.4 \%$ & $10.5 \%$ & $6.5 \%$ \\
\hline k__Bacteria;p__Firmicutes;c__Clostridia;o__Clostridiales;f__Lachnospiraceae;g__Dorea & $0.0 \%$ & $7.1 \%$ & $4.9 \%$ & $1.0 \%$ \\
\hline k__Bacteria;p__Firmicutes;c__Clostridia;o__Clostridiales;f__Lachnospiraceae;g__Lachnospira & $0.0 \%$ & $1.6 \%$ & $0.0 \%$ & $0.1 \%$ \\
\hline k__Bacteria;p_Firmicutes;c__Clostridia;o__Clostridiales;f_L_Lachnospiraceae;g__Roseburia & $0.0 \%$ & $0.1 \%$ & $21.1 \%$ & $3.3 \%$ \\
\hline k__Bacteria;p__Firmicutes;c__Clostridia;o__Clostridiales;f__Lachnospiraceae;g__Ruminococcus] & $0.0 \%$ & $6.7 \%$ & $1.0 \%$ & $6.0 \%$ \\
\hline k__Bacteria;p__Firmicutes;c__Clostridia;o__Clostridiales;f__Ruminococcaceae;Other & $0.0 \%$ & $0.0 \%$ & $0.2 \%$ & $2.9 \%$ \\
\hline k__Bacteria;p_Firmicutes;c__Clostridia;o__Clostridiales;f_Ruminococcaceae;g__Butyricicoccus & $0.0 \%$ & $0.7 \%$ & $0.4 \%$ & $0.2 \%$ \\
\hline k_Bacteria;p_Firmicutes;c__Clostridia;o__Clostridiales;f_Ruminococcaceae;g_Faecalibacterium & $0.0 \%$ & $0.1 \%$ & $0.4 \%$ & $3.8 \%$ \\
\hline k__Bacteria;p_Firmicutes;c__Clostridia;o__Clostridiales;f_Ruminococcaceae;g_Oscillospira & $0.0 \%$ & $0.7 \%$ & $2.4 \%$ & $3.8 \%$ \\
\hline k__Bacteria;p__Firmicutes;c__Clostridia;o__Clostridiales;f_Ruminococcaceae;g_Ruminococcus & $0.0 \%$ & $0.0 \%$ & $0.4 \%$ & $2.0 \%$ \\
\hline k_Bacteria;p_Firmicutes;c_Clostridia;o__Clostridiales;f_Veillonellaceae;g_Acidaminococcus & $0.0 \%$ & $0.6 \%$ & $0.0 \%$ & $0.0 \%$ \\
\hline k_Bacteria;p__Firmicutes;c_Clostridia;o__Clostridiales;f__Veillonellaceae;g__Megamonas & $0.0 \%$ & $15.1 \%$ & $0.0 \%$ & $0.0 \%$ \\
\hline k__Bacteria;p__Firmicutes;c__Clostridia;o__Clostridiales;f__Veillonellaceae;g__Megasphaera & $0.0 \%$ & $0.0 \%$ & $0.0 \%$ & $0.5 \%$ \\
\hline k__Bacteria;p_Firmicutes;c__Clostridia;o__Clostridiales;f__Veillonellaceae;g__Phascolarctobacterium & $0.0 \%$ & $0.9 \%$ & $2.9 \%$ & $3.7 \%$ \\
\hline k__Bacteria;p_Firmicutes;c__Clostridia;o__Clostridiales;f_Veillonellaceae;g_Veillonella & $9.1 \%$ & $1.2 \%$ & $0.0 \%$ & $0.0 \%$ \\
\hline k_Bacteria;p__Firmicutes;c__Clostridia;o__Clostridiales;f_[Mogibacteriaceae];g__ & $0.0 \%$ & $0.0 \%$ & $0.0 \%$ & $0.0 \%$ \\
\hline k__Bacteria;p__Firmicutes;c_Erysipelotrichi;o_Erysipelotrichales;f_Erysipelotrichaceae;Other & $5.7 \%$ & $0.9 \%$ & $0.2 \%$ & $0.0 \%$ \\
\hline k__Bacteria;p_Firmicutes;c__Erysipelotrichi;o__Erysipelotrichales;f_Erysipelotrichaceae;g__ & $0.0 \%$ & $0.0 \%$ & $0.0 \%$ & $2.7 \%$ \\
\hline k__Bacteria;p_Firmicutes;c__Erysipelotrichi;o__Erysipelotrichales;f_Erysipelotrichaceae;g__Coprobacillus & $0.0 \%$ & $0.0 \%$ & $3.3 \%$ & $0.0 \%$ \\
\hline k__Bacteria;p__Firmicutes;c_Erysipelotrichi;o__Erysipelotrichales;f_Erysipelotrichaceae;g_Holdemania & $0.0 \%$ & $0.0 \%$ & $0.2 \%$ & $0.0 \%$ \\
\hline k__Bacteria;p__Firmicutes;c__Erysipelotrichi;o_Erysipelotrichales;f__Erysipelotrichaceae;g_[Eubacterium] & $2.5 \%$ & $0.2 \%$ & $0.0 \%$ & $5.7 \%$ \\
\hline k__Bacteria;p__Firmicutes;c_Erysipelotrichi;o__Erysipelotrichales;f__Erysipelotrichaceae;g_cc_115 & $0.0 \%$ & $0.0 \%$ & $0.0 \%$ & $0.0 \%$ \\
\hline k__Bacteria;p__Fusobacteria;c__Fusobacteriia;o__Fusobacteriales;f_Fusobacteriaceae;Other & $0.0 \%$ & $3.3 \%$ & $0.4 \%$ & $0.5 \%$ \\
\hline k__Bacteria;p__Proteobacteria;c__Betaproteobacteria;o__Burkholderiales;f__Alcaligenaceae;g_Sutterella & $0.0 \%$ & $4.2 \%$ & $0.0 \%$ & $1.5 \%$ \\
\hline k__Bacteria;p__Proteobacteria;c__Deltaproteobacteria;o__Desulfovibrionales;f__Desulfovibrionaceae;g_Bilophila & $0.0 \%$ & $0.0 \%$ & $0.0 \%$ & $0.1 \%$ \\
\hline k__Bacteria;p_Proteobacteria;c__Gammaproteobacteria;o_Enterobacteriales;f_Enterobacteriaceae;g_Escherichia & $1.5 \%$ & $0.6 \%$ & $0.8 \%$ & $0.0 \%$ \\
\hline
\end{tabular}


the colonic microbiota than those involving the right part of the colon (7). A link has been suggested between metabolic diseases and bacterial populations in the gut. Ileum microbiota dysbiosis impairs the GLP-1-induced nitric oxide (NO) production by enteric neurons, which prevents the efficient activation of the gut-brain-to-periphery axis for the control of insulin secretion. Certain peptidoglycans from Lactobacillaceae may activate nucleotide-binding and oligomerization domain2/toll-like receptor4/CD14 to produce NO, resulting in enhanced GLP-1 action (8). Lactobacilli are positively and Porphyromonadaceae negatively correlated with the ileum GLP-1R and neuronal NO synthase mRNA expression (8). In this context, Although GLP-1RA sensitivity may be positively controlled by Lactobacillaceae and negatively by Porphyromonadaceae, no particular relevance was found in our study.

The absence of microbiota prevented GLP-1-induced insulin secretion, demonstrating a strong GLP-1 resistance (8). The GLP-1R levels were shown to be reduced in a germfree environment, and $\beta$-cells were glucose-unresponsive in the absence of gut microbiota (8). Although the volume of gut microbiota was not examined in the present study, colectomy may have caused reduced numbers of gut microbiota, resulting in GLP-1 resistance. Certain microbiota compositions (i.e. decreased Firmicutes and increased Bacteriodetes and Bifidobacteria) may lead to improved metabolic efficacy. The altered gut microbiota stimulates the differential production of short-chain fatty acids, such as butyrate, that in turn promote GLP-1 secretion from L-cells to improve metabolic health and protect against obesity and diabetes (9). The populations of Firmicutes and Bacteroidetes were indistinguishable among the subjects (Table 2). The population of Bifidobacterium seemed greater in the patient than in the controls (Table 6), but the patient did not receive any metabolic benefits against the development of diabetes. In terms of GLP-1 resistance and GLP-1 production, the composition of gut microbiota in this patient may not have had any major effects on controlling glucose homeostasis.

Since we do not have colectomized non-diabetic controls or more patients with diabetes due to UC colectomy, the analysis of the gut microbiota is just observational but not conclusive. As the residual small intestine takes over the function and microenvironment of the resected colon, our observation in gut microbiota may not be directly related to the diabetic phenotype in this patient but instead represent a transition in colonization of the residual small intestine. Thus, changes in the gut microbiota may not have been involved in the development of diabetes in this case.

We encountered a clinical case of a patient who underwent total colectomy due to severe UC and developed diabetes with defects in GLP-1 and GIP production. He was initially successfully treated with GLP-1 RA. The later worsening of glucose control, represented by a reduced insulin secretory capacity, suggests the importance of GIP for the long-term maintenance of pancreatic $\beta$-cells.

The development of diabetes in this particular patient may have been due to the elimination of colon GLP-1-producing cells by colectomy, based on the spontaneously reduction in his GLP-1/GIP production capability. The short-term effectiveness of GLP-1RA treatment suggested that prolonged GIP loss might cause gradual pancreatic $\beta$-cell dysfunction. Our report may explain the importance of residual incretins for maintaining the pancreatic $\beta$-cell function. This case report provides additional insight into the spatiotemporal role of incretins of the intestinal system in the control of wholebody glucose homeostasis.

\section{Author's disclosure of potential Conflicts of Interest (COI).}

Eiichi Araki: Research funding, Astellas Pharma, AstraZeneca, MSD, Ono Pharmaceutical, Kyowa Hakko Kirin, Sanofi, Shionogi, Takeda, Daiichi Sankyo, Mitsubishi Tanabe, Novo Nordisk and Pfizer; Honoraria: Astellas Pharma, AstraZeneca, MSD, Ono Pharmaceutical, Kowa Pharmaceutical, Sanofi, Takeda, Mitsubishi Tanabe, Eli Lilly, Novo Nordisk and Taisho Pharma.

\section{References}

1. Seino Y, Fukushima M, Yabe D. GIP and GLP-1, the two incretin hormones: Similarities and differences. J Diabetes Investig 1: 8-23, 2010.

2. Seino Y, Yabe D. Glucose-dependent insulinotropic polypeptide and glucagon-like peptide-1: incretin actions beyond the pancreas. J Diabetes Investig 4: 108-130, 2013.

3. Tura A, Pacini G, Yamada Y, Seino Y, Ahren B. Glucagon and insulin secretion, insulin clearance, and fasting glucose in GIP receptor and GLP-1 receptor knockout mice. Am J Physiol Regul Integr Comp Physiol 316: R27-R37, 2019.

4. Hansotia T, Drucker DJ. GIP and GLP-1 as incretin hormones: lessons from single and double incretin receptor knockout mice. Regul Pept 128: 125-134, 2005.

5. Holst JJ. The incretin system in healthy humans: the role of GIP and GLP-1. Metabolism 96: 46-55, 2019.

6. Yamazaki H, Matsuoka K, Fernandez J, et al. Ulcerative colitis outcomes research in Japan: protocol for an observational prospective cohort study of YOURS (YOu and Ulcerative colitis: Registry and Social network). BMJ Open 9: e030134, 2019.

7. Jensen AB, Sorensen TI, Pedersen O, Jess T, Brunak S, Allin KH. Increase in clinically recorded type 2 diabetes after colectomy. Elife 7: e37420, 2018.

8. Grasset E, Puel A, Charpentier J, et al. A specific gut microbiota dysbiosis of type 2 diabetic mice induces GLP-1 resistance through an enteric NO-dependent and Gut-Brain axis mechanism. Cell Metab 25: 1075-1090 e1075, 2017.

9. Yadav H, Lee JH, Lloyd J, Walter P, Rane SG. Beneficial metabolic effects of a probiotic via butyrate-induced GLP-1 hormone secretion. J Biol Chem 288: 25088-25097, 2013.

10. Jorsal T, Rhee NA, Pedersen J, et al. Enteroendocrine $K$ and $L$ cells in healthy and type 2 diabetic individuals. Diabetologia 61: 284-294, 2018.

11. Palnaes Hansen C, Andreasen JJ, Holst JJ. The release of gastric inhibitory peptide, glucagon-like peptide-I, and insulin after oral glucose test in colectomized subjects. Scand J Gastroenterol 32: 473-477, 1997.

12. Duan L, Rao X, Braunstein Z, Toomey AC, Zhong J. Role of incretin axis in inflammatory bowel disease. Front Immunol 8: 1734, 2017.

13. de la Cuesta-Zuluaga J, Mueller NT, Corrales-Agudelo V, et al. 
Metformin is associated with higher relative abundance of mucindegrading akkermansia muciniphila and several short-chain fatty acid-producing microbiota in the gut. Diabetes Care 40: 54-62, 2017.

14. Usui R, Sakuramachi Y, Seino Y, et al. Reply to the comment of Wilbrink et al. on Retrospective analysis of liraglutide and basal insulin combination therapy in Japanese type 2 diabetes: the association between remaining beta-cell function and the achievement of the HbA1c target 1 year after initiation. J Diabetes Investig 9: 981-983, 2018.

15. Usui R, Yabe D, Kuwata H, Murotani K, Kurose T, Seino Y. Retrospective analysis of safety and efficacy of liraglutide monotherapy and sulfonylurea-combination therapy in Japanese type 2 diabetes: association of remaining beta-cell function and achievement of HbA1c target one year after initiation. J Diabetes Complications 29: 1203-1210, 2015.

16. Piccand J, Vagne $\mathrm{C}$, Blot $\mathrm{F}$, et al. Rfx6 promotes the differentiation of peptide-secreting enteroendocrine cells while repressing genetic programs controlling serotonin production. Mol Metab 29: 24-39, 2019.

17. Donaldson GP, Lee SM, Mazmanian SK. Gut biogeography of the bacterial microbiota. Nat Rev Microbiol 14: 20-32, 2016.

The Internal Medicine is an Open Access journal distributed under the Creative Commons Attribution-NonCommercial-NoDerivatives 4.0 International License. To view the details of this license, please visit (https://creativecommons.org/licenses/ by-nc-nd/4.0/).

(C) 2021 The Japanese Society of Internal Medicine Intern Med 60: 1433-1442, 2021 